\title{
Packing Density of the Erythropoietin Receptor Transmembrane Domain Correlates with Amplification of Biological Responses ${ }^{\dagger}$
}

\author{
Verena Becker, ${ }^{\ddagger}$ Durba Sengupta, ${ }^{\S, l}$ Robin Ketteler, ${ }^{\perp}$ G. Matthias Ullmann, ${ }^{\#}$ Jeremy C. Smith,,${ }^{\S, \diamond}$ and \\ Ursula Klingmüller*, \\ Division Systems Biology of Signal Transduction, DKFZ-ZMBH Alliance, German Cancer Research Center, Heidelberg, \\ Germany, Interdisciplinary Center for Scientific Computing, University of Heidelberg, Heidelberg, Germany, Center for \\ Computational and Integrative Biology, Massachusetts General Hospital, Boston, Massachusetts 02114, Structural Biology/ \\ Bioinformatics, University of Bayreuth, Bayreuth, Germany, and University of Tennessee/ORNL Center for Molecular \\ Biophysics, Oak Ridge National Laboratory, Oak Ridge, Tennessee 37831-6164
}

Received July 30, 2008; Revised Manuscript Received September 11, 2008

\begin{abstract}
The formation of signal-promoting dimeric or oligomeric receptor complexes at the cell surface is modulated by self-interaction of their transmembrane (TM) domains. To address the importance of TM domain packing density for receptor functionality, we examined a set of asparagine mutants in the TM domain of the erythropoietin receptor (EpoR). We identified EpoR-T242N as a receptor variant that is present at the cell surface similar to wild-type EpoR but lacks visible localization in vesicle-like structures and is impaired in efficient activation of specific signaling cascades. Analysis by a molecular modeling approach indicated an increased interhelical distance for the EpoR-T242N TM dimer. By employing the model, we designed additional mutants with increased or decreased packing volume and confirmed a correlation between packing volume and biological responsiveness. These results propose that the packing density of the TM domain provides a novel layer for fine-tuned regulation of signal transduction and cellular decisions.
\end{abstract}

Key mediators of cellular communication are cell surface receptors that bind extracellular ligands and trigger the activation of complex intracellular signaling networks. The transmembrane $(\mathrm{TM})^{1}$ domain not only anchors cell surface receptors in the plasma membrane but orientation and selfinteraction of the TM domain critically modulate the initiation of signal transduction (1). Receptor activating mutations of the TM domain have been reported for receptor tyrosine kinases $(2,3)$ as well as cytokine receptors $(4,5)$ and have been linked to disease progression (6). Cytokine receptors are single membrane-spanning proteins, and recent studies demonstrated the existence of preformed homodimers in the absence of ligand for receptors such as the erythropoietin receptor (EpoR) (7), the growth hormone receptor (8), and the leptin receptor (9). The EpoR is a prototype cytokine

\footnotetext{
${ }^{\dagger}$ This work was supported by the European Commission Sixth Framework Programme COSBICS project (LSHG-CT-2004-512060) (to V.B.) and by the Deutsche Forschungsgemeinschaft (DFG/Ke904) (to R.K.).

* Corresponding author: tel, 49-6221-42 4481; fax, 49-6221-42 4488; e-mail, u.klingmueller@dkfz-heidelberg.de.

ॠ German Cancer Research Center.

${ }^{\S}$ University of Heidelberg.

"Present address: Department of Biophysical Chemistry, University of Groningen, Groningen, The Netherlands.

${ }^{\perp}$ Massachusetts General Hospital.

\# University of Bayreuth.

${ }^{\diamond}$ University of Tennessee.

${ }^{1}$ Abbreviations: TM, transmembrane; EpoR, erythropoietin receptor; JAK, Janus kinase; STAT, signal transducer and activator of transcription; MAPK, mitogen-activated protein kinase; PI3K, phosphatidylinositol 3-kinase; GFP, green fluorescent protein; ERK, extracellular signal-regulated kinase; Akt/PKB, protein kinase B; CFU-E, colony forming unit-erythroid.
}

receptor and key regulator of erythropoiesis (10). It has been reported that self-assembly of the EpoR TM domain critically promotes receptor activation in response to ligand binding $(11,12)$.

Molecular modeling approaches performed in vacuum have been used to obtain information about the interhelical interfaces of TM dimers of the receptor tyrosine kinase ErbB2 $(13,14)$ as well as the EpoR (15). However, to elucidate the interaction of the TM domain with the surrounding lipids as well as the packing density of helix-helix pairs requires a modeling approach taking into account the membranous context. A molecular model distinguishing between the solvent, headgroup, and core regions of a membrane has been successfully applied to reproduce the experimentally determined tilt angle of glycophorin A (16). Therefore, such an approach could be very useful to provide essential structural information on the EpoR TM dimers. Deregulation of EpoR signaling may result in erythroleukemia or more frequently anemia, thus requiring specific intervention. To access the TM domain of the EpoR by a rational design of therapeutic components in analogy to the study of integrins (17), a deeper understanding of structural and mechanistic characteristics of TM helices is required due to the versatile effects of the TM domain on receptor signaling.

Facilitated by the TM domain, the EpoR undergoes a conformational switch upon ligand binding, which subsequently leads to activation of the receptor-associated cytoplasmic Janus kinase JAK2 (18). Activated JAK2 phosphorylates tyrosine residues within the EpoR cytoplasmic domain that serve as docking sites for signal-transducing molecules, 
triggering downstream signaling cascades including the signal transducer and activator of transcription STAT5 and the mitogen-activated protein kinase (MAPK) as well as the phosphatidylinositol 3-kinase (PI3K) pathway (19). Even though signal transduction through the EpoR has been studied in detail, it remains unresolved if specific properties of the TM domain interaction permit selective amplification of specific signaling cascades and thereby fine-tuning of biological responses.

In this study, we show by combining a molecular modeling approach in an implicit membrane environment with functional studies that high packing density of the EpoR TM domain correlates with an intracellular detection in vesiclelike structures and amplification of selective signaling pathways as well as biological responsiveness.

\section{MATERIALS AND METHODS}

Cells. Phoenix eco and BaF3 cells were cultured as described (20). NIH3T3 cells were cultured in DMEM supplemented with $10 \%$ calf serum (Invitrogen). Fetal liver cells from Balb/c EpoR ${ }^{-1-}$ mice (provided by $\mathrm{H}$. Wu, University of California, Los Angeles) were prepared from d12.5 embryos. Transfections were performed by calcium phosphate precipitation as described (20). Transducing supernatants were generated $24 \mathrm{~h}$ after transfection, supplemented with $8 \mu \mathrm{g} / \mathrm{mL}$ Polybrene. BaF3 cells expressing EpoR variants were selected in $1.5 \mu \mathrm{g} / \mathrm{mL}$ puromycin $48 \mathrm{~h}$ after transduction. NIH3T3 cells were selected in $3 \mu \mathrm{g} / \mathrm{mL}$ puromycin $24 \mathrm{~h}$ after transduction, and cells expressing Rab proteins were additionally selected in $400 \mu \mathrm{g} / \mathrm{mL}$ G4 18 .

Plasmids. Retroviral expression vectors were pMOWSpuro or pMOWS-neo (20). For details on cloning refer to the Supporting Information.

Flow Cytometry. To analyze cell surface expression, BaF3 cells expressing HA-tagged EpoR-GFP chimeric proteins were stained as described (21) and analyzed by flow cytometry using a FACSCalibur (Becton Dickinson). Live cells were gated by forward and side scatter, and the mean fluorescence intensity (MFI) is given as the percentage of MFI of wild-type EpoR. To analyze apoptosis, BaF3 cells expressing HA-tagged wild-type EpoR or EpoR-T242N were washed with RPMI 1640, seeded at a density of $4 \times 10^{4}$ cells/mL, and grown in 1 unit/mL Epo (Janssen-Cilag). Cells were counted 24,48 , and $72 \mathrm{~h}$ later, and $3 \times 10^{5}$ cells were stained with annexin V-PE and 7-AAD and analyzed using a FACSCalibur. Annexin V-PE positive and 7-AAD negative cells were regarded as apoptotic cells. Cells grown in RPMI 1640 without Epo or in medium supplemented with 5\% WEHI supernatant as a source of IL-3 were taken as control.

Fluorescence Microscopy. NIH3T3 cells were subcultivated on chambered coverglass (Nalge Nunc). Analysis of GFP-tagged wild-type HA-EpoR and HA-EpoR mutants was performed on a Nikon Eclipse TE2000 inverted microscope with a Perkin-Elmer UltraView ERS spinning disk confocal unit on a heated stage $\left(37^{\circ} \mathrm{C}\right)$ and a Nikon Plan Apo $60 \times /$ 1.2 WI objective. Images were acquired with a Hamamatsu EM-CCD camera and UltraView ERS Imaging Suite software. Adjustments of brightness and contrast were performed using Adobe Photoshop 7.0.

Metabolic Labeling. BaF3 cells expressing HA-tagged wild-type EpoR or EpoR-T242N were washed with cysteine- and methionine-free RPMI (Invitrogen) and preincubated for $45 \mathrm{~min}$ at $37{ }^{\circ} \mathrm{C}$ in Cys/Met-free RPMI including $10 \%$ dialyzed FCS (Invitrogen). Labeling was performed with 0.5 $\mathrm{mCi}$ of ${ }^{35} \mathrm{~S}$ in vitro cell labeling mix (GE Healthcare)/ $1 \times$ $10^{7}$ cells for $15 \mathrm{~min}$ at $37^{\circ} \mathrm{C}$. Subsequently, cells were chased by adding an excess of unlabeled cysteine and methionine. For each time point, $1 \times 10^{6}$ cells were lysed in RIPA buffer (50 mM Tris, $\mathrm{pH} 7.6,150 \mathrm{mM} \mathrm{NaCl}, 1 \% \mathrm{NP}-40,0.5 \%$ sodium deoxycholate, $0.1 \%$ SDS) supplemented with aprotinin and AEBSF. Immunoprecipitates were digested with EndoH for $1 \mathrm{~h}$ at $37^{\circ} \mathrm{C}$ or left untreated, separated by $10 \%$ SDS-PAGE, transferred to a nitrocellulose membrane, and detected on a Typhoon phosphoimager (GE Healthcare). Signals were quantified with LumiAnalyst 3.1 software (Roche Diagnostics).

Receptor Internalization. $\mathrm{BaF} 3$ cells $\left(1 \times 10^{6}\right)$ expressing GFP-tagged wild-type HA-EpoR or HA-EpoR-T242N were starved in RPMI 1640 supplemented with $1 \mathrm{mg} / \mathrm{mL}$ BSA for $1 \mathrm{~h}$ and stimulated with $500 \mathrm{pM}\left[{ }^{125} \mathrm{I}\right]-\mathrm{Epo}$ (GE Healthcare) for the indicated time at $37^{\circ} \mathrm{C}$. Subsequently, cells were immediately transferred to ice, and free $\left[{ }^{125} \mathrm{I}\right]-$ Epo was separated from the cells by centrifugation through a layer of FCS. Cell surface-bound [ $\left.{ }^{125} \mathrm{I}\right]$-Epo was stripped by incubation with $4 \%$ acetic acid for $5 \mathrm{~min}$ on ice and subsequent centrifugation through FCS. The efficiency of acid stripping was approximately 95\%, and membrane integrity after acid stripping was confirmed by Trypan blue exclusion. Samples were measured in a $\gamma$ counter. Specific binding was determined by subtracting values obtained with an excess of unlabeled Epo (Janssen-Cilag).

Proliferation Assay. Proliferation assays of BaF3 cells stably expressing GFP-tagged wild-type HA-EpoR or HAEpoR TM domain mutants were performed as described (22). Results are expressed as cell number/mL.

CFU-E Colony Assay. CFU-Es derived from d12.5 EpoR $^{-1-}$ embryos were transduced with diluted supernatants, plated in $0.8 \%$ methylcellulose (StemCell Technologies) supplemented with 4 units/mL Epo (Janssen-Cilag) and 20 $\mathrm{ng} / \mathrm{mL}$ SCF (R\&D Systems), and stained with $0.04 \%$ benzidine after 2 days.

Immunoprecipitation and Immunoblotting. BaF3 $\left(1 \times 10^{7}\right)$ cells were starved in RPMI 1640 with $1 \mathrm{mg} / \mathrm{mL}$ BSA for $3 \mathrm{~h}$, stimulated with Epo (Janssen-Cilag) for $10 \mathrm{~min}$ at 37 ${ }^{\circ} \mathrm{C}$, and lysed with NP-40 lysis buffer (1\% NP-40, $150 \mathrm{mM}$ $\mathrm{NaCl}, 20 \mathrm{mM}$ Tris, $\mathrm{pH}$ 7.4, $10 \mathrm{mM}$ NaF, 1 mM EDTA, $\mathrm{pH}$ 8.0, $1 \mathrm{mM} \mathrm{ZnCl}_{2}, \mathrm{pH} 4.0,1 \mathrm{mM} \mathrm{MgCl}_{2}, 1 \mathrm{mM} \mathrm{Na}_{3} \mathrm{VO}_{4}$, $10 \%$ glycerol) supplemented with aprotinin and AEBSF. Immunoprecipitates were performed using anti-EpoR (Santa Cruz, M-20), anti-JAK2 (Upstate), or anti-STAT5 (Santa Cruz, C-17) antibodies, separated by $10 \%$ SDS-PAGE, and transferred to a nitrocellulose membrane. For analysis of ERK1/2 and Akt/PKB, $100 \mu \mathrm{g}$ of cellular lysates were separated by $15 \%$ SDS-PAGE and transferred to a PVDF membrane. Analysis of immunoblots was performed using anti-phosphotyrosine clone 4G10 (Upstate), anti-phosphoAkt (Ser 473) (Cell Signaling Technology), or anti-phosphoERK1/2 (Thr 202/ Tyr 204) (Cell Signaling Technology) antibodies. To remove antibodies for analysis of total protein, membranes were treated with SDS and $\beta$-mercaptoethanol (23) and incubated with anti-EpoR, anti-JAK2, anti-STAT5, anti-ERK1/2 (Cell Signaling Technology), or anti-Akt (Cell Signaling Technology) antibodies. Secondary antibodies were 
anti-mouse (for phosphorylated immunoprecipitated proteins) or anti-rabbit antibodies (for cellular lysates) or protein A (for total immunoprecipitated proteins) coupled to horseradish peroxidase (GE Healthcare). Detection was performed with enhanced chemiluminescence (GE Healthcare) and a charge-coupled device camera using the LumiImager (Roche). Immunoblots were quantified with LumiAnalyst 3.1 software. Relative values were scaled to 1 and plotted as arbitrary units. For time course analysis, a smoothing spline was plotted through the mean of three independent data sets. For dosedependence analysis, a sigmoidal plot was fitted to five independent data sets (pSTAT5) or three data sets (pERK1, $\mathrm{pAkt} / \mathrm{PKB})$.

All-Atom Structures of the EpoR TM Dimer Modeled in a Membrane Environment. The most probable structure of a transmembrane dimer is that with the lowest solvation energy, $\Delta G_{\text {solv }}$, defined as the free energy change for transferring the molecule from vacuum to its position in the membrane (24). The solvation energy was calculated using an all-atom model for the EpoR transmembrane dimer together with the Poisson-Boltzmann equation for the electrostatic solvation energy and a term representing the cost of cavity formation in the aqueous solvent as described (16). A five-slab continuum dielectric model that distinguishes between the solvent, headgroup, and core regions was used to describe the membrane environment. For a dimer in the membrane, the structure was described by four parameters: the interhelical distance $d$, the crossover angle $\theta$, the membrane insertion $v$, and helix rotation $r . d$ is defined as the minimum distance between the two helix axes and $\theta$ the angle between them. $v$ is the distance of the center of mass of each helix from the center of the membrane. $r$ is the angle of rotation of each helix about its axis. The transmembrane segment of EpoR (LILTLSLILVLISLLLTV LALLS) was modeled as an ideal $\alpha$-helix with $\psi=-47^{\circ}$ and $\phi=-57^{\circ}$ (25). Values were assigned to $d, \theta, v$, and $r$ systematically so as to sample the relevant conformational space of the homodimer. The side chains were positioned using the software SCWRL (26) (version 3.0), which determines the most probable rotameric conformations from a backbone-dependent rotamer library by an algorithm-based graph theory. The dimers were energy-minimized in a dielectric medium of $\varepsilon=2$ using the CHARMM potential (27) (version 30b1) with 1000 steps of steepest descent followed by 1000 steps of Newton-Raphson minimization with $1 \mathrm{kcal} / \mathrm{mol}$ harmonic constraints on the backbone atoms. The structures were then introduced in the five-slab membrane model and scored based on the value of the solvation energy. The volume of a dimer was calculated as the volume defined by rolling a sphere of radius $2.2 \AA$ on the molecular surface to fill all cavities in the dimer interface. The lowest energy conformers along with the conformers with energies accessible at $300 \mathrm{~K}\left(K_{\mathrm{B}} T\right)$ were chosen. The free energy of dimerization of the dimer conformations was negative in all cases, implying that the EpoR dimers do indeed form.

\section{RESULTS}

Mutations in the EpoR TM Domain Affect Cell Surface Expression and Detection in Vesicular Structures. To address the effect of enhanced self-interaction of the TM domain for EpoR signaling, we examined seven asparagine TM
A

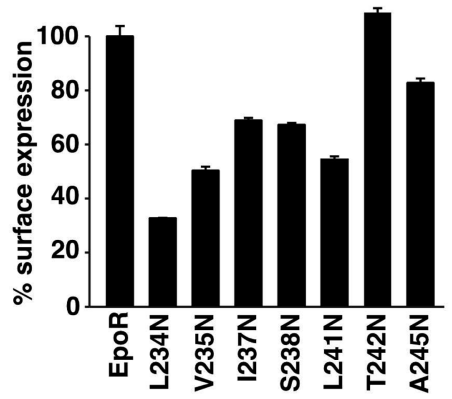

B
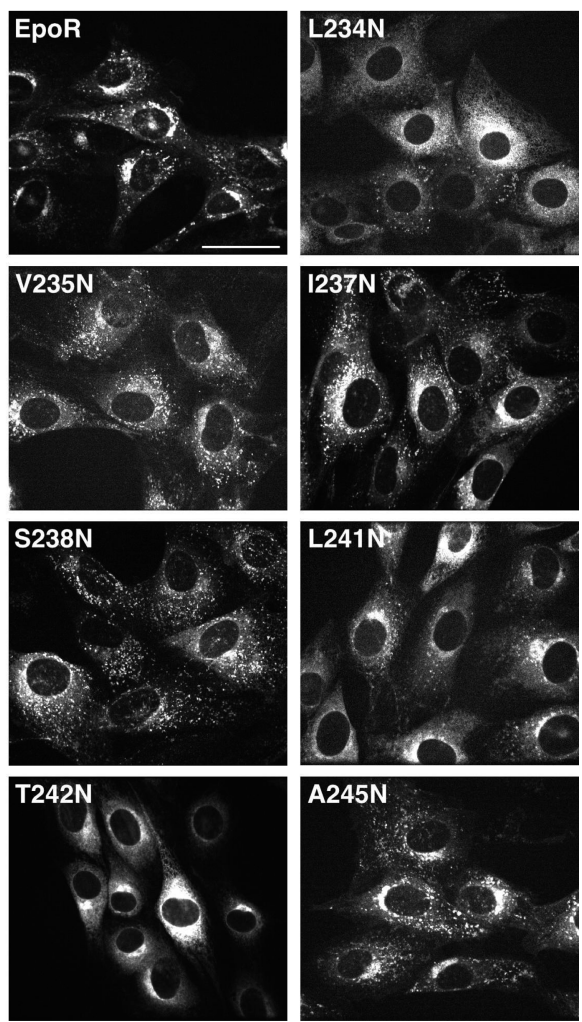

A245N

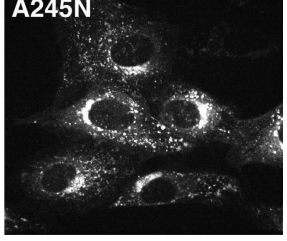

FIGURE 1: Flow cytometry analysis and live cell imaging of GFPtagged EpoR TM domain mutants. (A) Cell surface expression of GFP-tagged wild-type HA-EpoR and HA-EpoR mutants in stably transduced $\mathrm{BaF} 3$ cells was assessed by flow cytometry and is shown as the percentage of the wild-type EpoR (mean $\pm \mathrm{SD}, n=3$ ). (B) Expression of GFP-tagged wild-type HA-EpoR or HA-EpoR mutants in NIH3T3 cells was assessed by spinning disk confocal microscopy, and representative images are shown. Scale bar is 20 $\mu \mathrm{m}$.

domain mutants, L234N, V235N, I237N, S238N, L241N, $\mathrm{T} 242 \mathrm{~N}$, and A245N, within the context of the full-length EpoR. These mutations were chosen based on their capacity to increase self-assembly in a bacterial ToxR reporter assay (22). As evidenced by flow cytometry (Figure 1A), the majority of these mutant receptors were expressed at significantly reduced levels at the plasma membrane of the murine hematopoietic cell line BaF3. Only EpoR-T242N and EpoR-A245N showed cell surface expression comparable to wild-type EpoR or a decrease by less than $20 \%$, respectively. To examine the subcellular localization, green fluorescent protein- (GFP-) tagged wild-type EpoR and EpoR TM domain mutants were expressed in fibroblast NIH3T3 cells (Figure 1B). Similar to wild-type EpoR, expression of GFPtagged EpoR mutants A245N as well as L234N, V235N, I237N, S238N, and L241N resulted in a vesicle-like localization pattern, partially colocalizing with markers for early and 
A
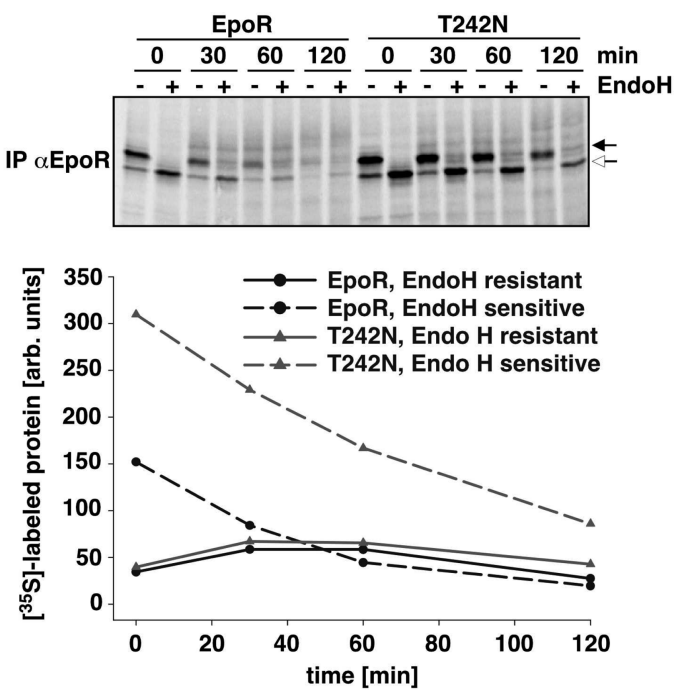

B

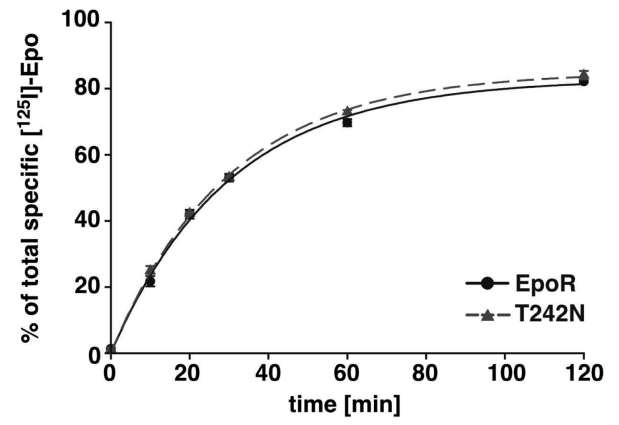

FIGURE 2: Maturation and internalization kinetics of wild-type EpoR and EpoR-T242N. (A) BaF3 cells stably expressing HA-tagged wild-type EpoR or EpoR-T242N were labeled with $\left[{ }^{35}\right.$ S $]$ methionine/ cysteine and chased for up to $2 \mathrm{~h}$. After immunoprecipitation with anti-EpoR antibodies, immunoprecipitates were left untreated or digested with EndoH for $1 \mathrm{~h}$. Proteins were separated by SDS-PAGE, transferred to a nitrocellulose membrane, and detected on a phosphoimager. Closed and open arrows indicate the glycosylated and unglycosylated EpoR, respectively. The experiment was performed three times, and a representative analysis is shown (upper panel). Levels of ${ }^{35} \mathrm{~S}$-labeled proteins of the EndoH-resistant as well as EndoH-sensitive receptor proteins for wild-type EpoR or EpoR$\mathrm{T} 242 \mathrm{~N}$ were quantified and expressed in arbitrary units (lower panel). (B) BaF3 cells stably expressing GFP-tagged wild-type HAEpoR or HA-EpoR-T242N were starved and subsequently labeled with $\left[{ }^{125} \mathrm{I}\right]$-Epo for the indicated time. After separation of free ligand and acid stripping, cell surface-bound and internalized $\left[{ }^{125} \mathrm{I}\right]$-Epo were measured, and internalized ligand was plotted as the percentage (mean $\pm \mathrm{SD}$ ) of the total of cell surface and internalized radioactivity for three independent data sets.

recycling endosomes Rab4a and Rab5a as shown for wildtype EpoR (Figure S1 of the Supporting Information). Surprisingly, the mutant EpoR-T242N exhibited a pattern indicative of primarily endoplasmic reticulum and Golgi localization, and vesicle-like structures were not detected (Figure 1B) even after ligand stimulation (data not shown).

EpoR-T242N Maturation and Internalization Are Comparable to Wild-Type EpoR. To examine transport of EpoR$\mathrm{T} 242 \mathrm{~N}$ to and removal from the cell surface, we analyzed processing and internalization kinetics of EpoR-T242N in detail. Receptor processing was determined in BaF3-EpoR and BaF3-EpoR-T242N cells that were pulse-labeled with $\left.{ }^{[35} \mathrm{S}\right] \mathrm{methionine} / \mathrm{cysteine}$ and chased for up to $2 \mathrm{~h}$. For each time point, immunoprecipitated EpoR was digested with EndoH or left untreated (Figure 2A, upper panel). For samples not digested with EndoH, quantification of protein levels revealed a half-life of $36 \pm 3$ min for wild-type EpoR, whereas EpoR-T242N revealed a significantly longer halflife of $103 \pm 11$ min (mean $\pm \mathrm{SD}, n=3$ ). An increase in half-life is also observed for EndoH-sensitive EpoR-T242N (unglycosylated form, EndoH-digested samples), which may give rise to higher signals for total as well as EndoH-sensitive receptor protein for EpoR-T242N at all time points observed. In addition, this increased half-life of EndoH-sensitive EpoRT242N might contribute to the prominent endoplasmic reticulum staining as observed by live cell imaging in NIH3T3 cells (Figure 1B). In line with comparable cell surface expression levels (Figure 1A), the EndoH-resistant form of both wild-type EpoR and EpoR-T242N (glycosylated form, EndoH-digested samples) showed similar protein levels and kinetics (Figure 2A, lower panel), thereby indicating that cell surface transport of mature glycosylated receptor is comparable for these receptor variants.

Even after ligand stimulation, receptor-containing endosomes were not detectable in NIH3T3 cells expressing EpoRT242N (data not shown). Therefore, we asked whether this mutant is able to internalize upon Epo binding. BaF3 cells expressing wild-type EpoR or EpoR-T242N were starved and subsequently incubated with $\left.{ }^{125} \mathrm{I}\right]$-Epo for up to $120 \mathrm{~min}$. After separation of unbound ligand, cell surface-bound $\left[{ }^{125} \mathrm{I}\right]-$ Epo and internalized $\left[{ }^{125} \mathrm{I}\right]$-Epo were separated by acid stripping, and the amount of internalized ligand was expressed as the percentage of total cell-associated radioactivity. This analysis revealed that the EpoR-T242N showed internalization kinetics comparable to wild-type EpoR (Figure 2B) with $50 \%$ of the receptor internalized after approximately 25 min. Furthermore, total amounts of cell surface-bound [ $\left.{ }^{125} \mathrm{I}\right]$-Epo at each time point of the time course analysis are comparable for wild-type EpoR and EpoR-T242N (Figure S2 of the Supporting Information). Taking into account similar levels of cell surface expression (Figure 1A), this analysis shows that the ligand binding affinity for EpoR$\mathrm{T} 242 \mathrm{~N}$ is not altered compared to wild-type EpoR.

Thus, although vesicle-like structures could not be detected for EpoR-T242N, this mutant is fully capable of undergoing maturation, ligand binding, and internalization with kinetics similar to that of wild-type EpoR.

Altered Subcellular Detection of EpoR TM Mutant Correlates with an Impaired Capacity To Activate ERKI/2 and Akt/PKB Signaling. To examine the effect of the TM domain mutations on the activation of signal transduction, we focused our studies on the analysis of EpoR-T242N and EpoRA245N since both receptor variants showed cell surface expression comparable to wild-type EpoR but differed with respect to the detection in vesicular structures. By immunoblotting experiments, dose-dependent activation of the receptor and the associated kinase JAK2 was examined in $\mathrm{BaF} 3$ cells stably expressing wild-type EpoR or the mutant EpoR-T242N or EpoR-A245N. In line with metabolic labeling studies, an overall increased expression level of mutant EpoR-T242N and EpoR-A245N in comparison to wild-type EpoR was consistently detected (Figure 3). Whereas for wild-type EpoR and EpoR-A245N a proportional increase in EpoR and JAK2 tyrosine phosphorylation was observed, for EpoR-T242N levels of EpoR and JAK2 phosphorylation were significantly reduced even at high Epo concentrations (Figure 3). 


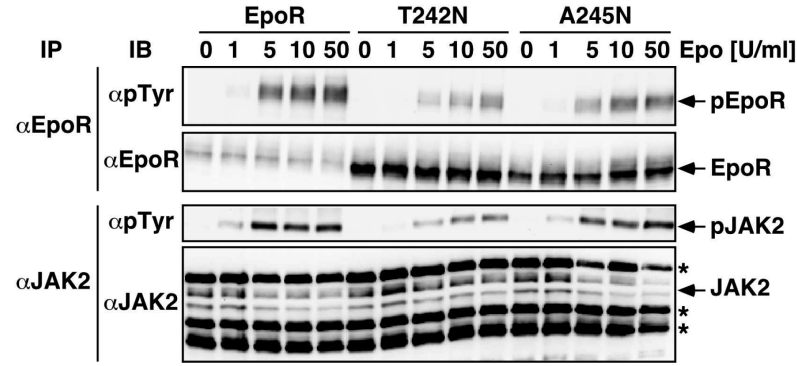

FIGURE 3: Immunoblot analysis of signal initiation through EpoRT242N. BaF3 cells stably expressing GFP-tagged wild-type HAEpoR or HA-EpoR TM domain mutants were stimulated with increasing concentrations of Epo for $10 \mathrm{~min}$. Lysates were subjected to immunoprecipitation (IP) with anti-EpoR and subsequently antiJAK2 antibodies. Immunoblots (IB) were analyzed with antiphosphotyrosine (pTyr) antibody and reprobed with anti-EpoR or anti-JAK2 antibodies. The experiment was performed three times, and a representative immunoblot analysis is shown. The asterisks indicate unspecific bands.

To investigate how impaired signal initiation through EpoR-T242N influences downstream pathways, we analyzed activation of STAT5 and extracellular signal-regulated kinases ERK1/2 as well as the protein kinase B (Akt/PKB) by quantitative immunoblotting (Figure 4). A sigmoidal plot was fitted to five independent data sets (pSTAT5) or three data sets (pERK1, pAkt/PKB). Whereas at low Epo concentrations, STAT5 tyrosine phosphorylation was decreased in BaF3-EpoR-T242N cells compared to BaF3 cells expressing wild-type EpoR, activation of STAT5 was comparable for wild-type EpoR and EpoR-T242N at elevated Epo levels (Figure 4A and 4B, upper panel). In general, signal transduction is not necessarily linear within a cascade of signaling molecules but may display amplification steps. This could explain the observation that STAT5 is more efficiently activated through EpoR-T242N compared to the upstream kinase JAK2 in BaF3-EpoR-T242N cells, showing saturation of STAT5 phosphorylation (Figure 4B, upper panel) for Epo concentrations at which JAK2 (Figure 3) is not yet saturated. Reprobing of the immunoblot showed variable levels of total STAT5 in the different cell lines. However, analyzing total STAT5 of five different data sets revealed no major difference for STAT5 levels of wild-type EpoR and EpoR-T242N (data not shown). In contrast to STAT5 activation, in EpoRT242N expressing BaF3 cells phosphorylation of ERK1/2 as well as Akt/PKB was dramatically reduced (Figure 4A and $4 \mathrm{~B}$, lower panels).

To investigate if activation of ERK1/2 and Akt/PKB through EpoR-T242N was delayed compared to wild-type EpoR, BaF3 cells expressing wild-type EpoR or EpoRT242N were stimulated with 50 units $/ \mathrm{mL}$ Epo for different periods up to $60 \mathrm{~min}$. Quantitative analysis of activated EpoR, ERK1, and Akt/PKB was performed by plotting a smoothing spline through the mean of three independent data sets. Analysis of phosphorylation revealed peak levels for receptor as well as ERK1 and Akt/PKB for wild-type EpoR after 5-10 min of Epo stimulation (Figure 5). In contrast, receptor activation as well as activation of ERK1 and Akt/ $\mathrm{PKB}$ in $\mathrm{BaF} 3$ cells expressing EpoR-T242N was delayed in comparison to wild-type EpoR, and maximum phosphorylation levels were obtained at 10 and $15 \mathrm{~min}$ of Epo stimulation, respectively (Figure 5). Despite elevated Epo concentration, Akt/PKB activation through EpoR-T242N was drastically reduced in comparison to wild-type EpoR expressing $\mathrm{BaF} 3$ cells (Figure 5B, lower right panel). Thus, signal transduction of EpoR-T242N through ERK1/2 and Akt/PKB shows delayed kinetics compared to wild-type EpoR.

Furthermore, coimmunoprecipitation experiments revealed that EpoR-T242N is capable of associating with various signaling molecules including the kinase JAK2 as well as the tyrosine phosphatase SHP-1 comparable to wild-type EpoR (Figure S3 of the Supporting Information). Thus, the deficiency in amplification of ERK1/2 and Akt/PKB signaling by the mutant EpoR-T242N is unlikely to be caused by altered interaction with these key molecules of EpoR signaling.

Reduced Capacity of EpoR-T242N To Support Proliferation and Differentiation. Next, we examined whether a reduced capacity to amplify certain signaling pathways results in deficiencies in stimulating biological responses. To compare proliferative responses, BaF3 cells expressing wild-type EpoR or EpoR-T242N were seeded at a density of $4 \times 10^{4}$ cells $/ \mathrm{mL}$ and grown in $1 \mathrm{unit} / \mathrm{mL}$ Epo for 3 days. While BaF3-EpoR cells reached up to $(92 \pm 4) \times 10^{4}$ cells/ $\mathrm{mL}, \mathrm{BaF} 3-\mathrm{EpoR}-\mathrm{T} 242 \mathrm{~N}$ cells only reached $(21 \pm 4) \times 10^{4}$ cells $/ \mathrm{mL}$ after 3 days of culture (Figure 6A, upper panel). To determine whether the reduced capacity of EpoR-T242N to support proliferation of $\mathrm{BaF} 3$ cells was due to an increase in apoptosis, the fraction of apoptotic cells was analyzed as the number of annexin $\mathrm{V}$-positive and 7-AAD-negative cells, and a representative overlay for day 3 is shown (Figure 6A, lower panel). This analysis revealed that the fraction of apoptotic cells was low and remained comparable for BaF3 cells expressing wild-type EpoR and EpoR-T242N as indicated by $(1.6 \pm 0.5) \%$ and $(2.2 \pm 0.4) \%$ apoptotic cells, respectively. Furthermore, the defect in proliferative responses observed for BaF3-EpoR-T242N cells could not be compensated by higher Epo concentrations (refer to Figure 9B). Thus, in line with efficient STAT5 activation, the reduction in cell numbers obtained for BaF3-EpoR-T242N cells in comparison to BaF3-EpoR cells is not due to accelerated apoptosis but to a decrease in cell proliferation.

To address the consequences of inefficient signal activation for expansion and differentiation of erythroid progenitor cells, we transduced fetal liver cells from d12.5 $\mathrm{EpoR}^{-1-}$ embryos to reconstitute the expression of wild-type EpoR or mutant EpoR-T242N (Figure 6B). As expected, mock-transduced fetal liver cells formed no CFU-E colonies, whereas expression of wild-type EpoR supported the formation of $172 \pm$ 27 colonies. In contrast, transduction of EpoR-T242N resulted in a significantly reduced number of $72 \pm 11 \mathrm{CFU}-\mathrm{E}$ colonies ( $p>0.005$ compared to wild-type EpoR as revealed by a two-sided unpaired Student's $t$ test), suggesting that maximal proliferation and differentiation of erythroid progenitor cells depend on efficient activation of ERK1/2 and Akt/PKB signaling.

Thus, distinct from EpoR-A245N (Figure S4 of the Supporting Information), EpoR-T242N is expressed at the cell surface comparable to the wild-type receptor but not detectable in vesicle-like structures and impaired in eliciting biological responses.

Molecular Modeling Shows Increased Packing Volume for Mutant EpoR Dimers. To gain insight into molecular mechanisms underlying the severe deficiencies of EpoR- 
B

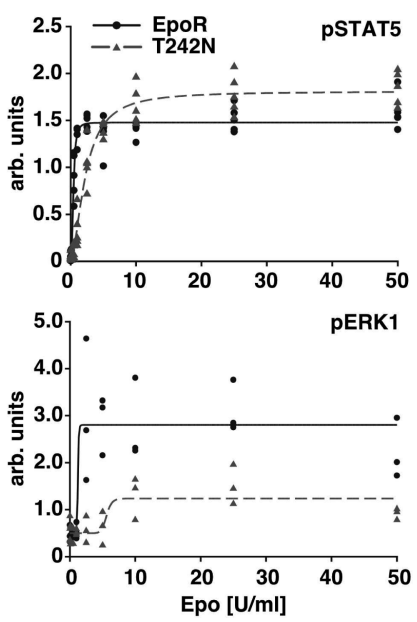

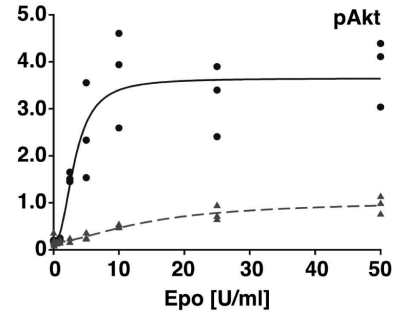

FIgURE 4: Immunoblot analysis of ERK1/2 and Akt/PKB activation through EpoR-T242N. (A) BaF3 cells stably expressing HA-tagged wild-type EpoR or EpoR-T242N were stimulated with increasing concentrations of Epo. Cellular lysates (CL) were immunoblotted (IB) with anti-phospho-Akt, anti-phospho-ERK, anti-Akt, and anti-ERK antibodies. The rest of the lysate was subjected to immunoprecipitation (IP) with anti-STAT5 antibodies, and samples were analyzed by immunoblotting with anti-phosphotyrosine (pTyr) and anti-STAT5 antibodies. Immunoblot signals were acquired using a charge-coupled device camera, and a representative immunoblot analysis is shown. (B) Phosphorylation levels of STAT5, ERK1, and Akt/PKB were analyzed by Lumilmager quantification. A sigmoidal curve was fitted through arbitrary units representing five independent data sets (pSTAT5) or three independent data sets (pERK1, pAkt).

A

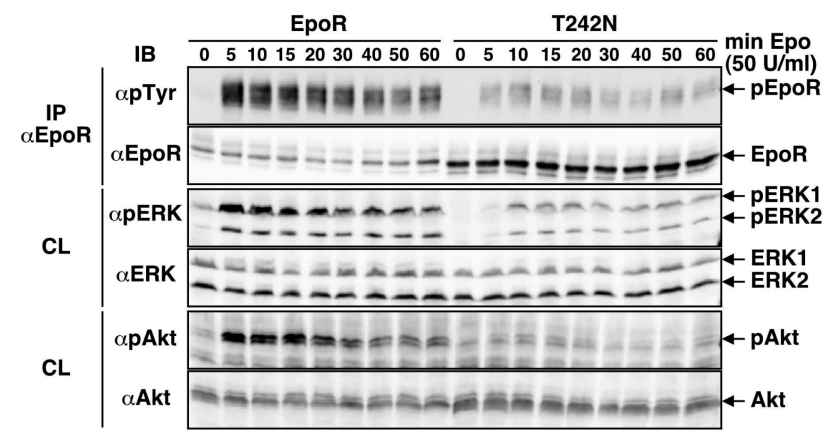

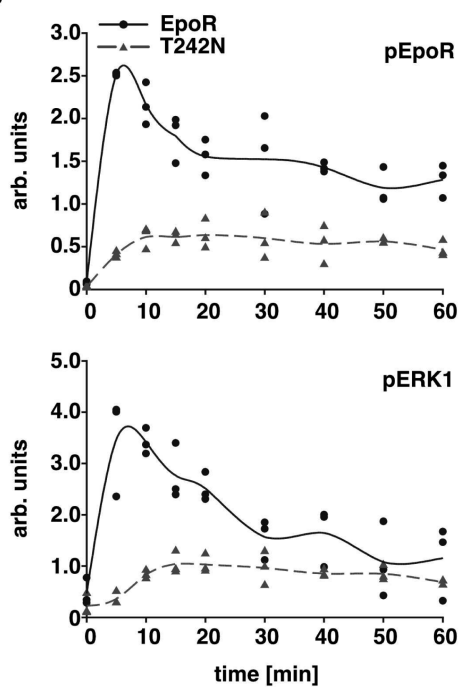

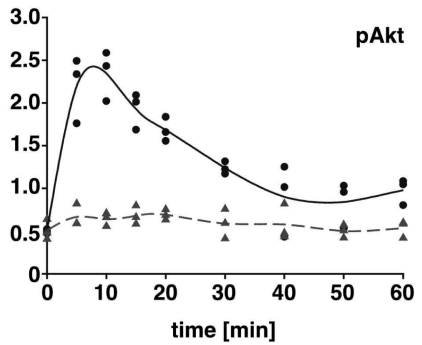

FIGURE 5: Kinetic analysis of ERK and Akt/PKB activation through EpoR-T242N. (A) BaF3 cells stably expressing HA-tagged wild-type EpoR or EpoR-T242N were stimulated with 50 units $/ \mathrm{mL}$ Epo for different periods up to 60 min. The cellular lysates (CL) were immunoblotted (IB) with anti-phospho-Akt, anti-phospho-ERK, anti-Akt, and anti-ERK antibodies. The rest of the lysate was subjected to immunoprecipitation (IP) with anti-EpoR antibodies. The immunoprecipitates were analyzed with anti-phosphotyrosine (pTyr) and anti-EpoR antibodies. Immunoblot signals were acquired using a charge-coupled device camera, and a representative immunoblot analysis is shown. (B) Phosphorylation levels of EpoR, ERK1, and Akt/PKB were analyzed by LumiImager quantification of the respective immunoblots. A smoothing spline was plotted through the mean of arbitrary units of three independent data sets.

T242N, we modeled the structures of EpoR TM dimers in atomic detail in a membrane environment. The modeled structure of the wild-type EpoR TM dimer exhibited two low-energy interfaces of left-handed helix-helix pairs (Figure 7A). The model for the lowest energy structure I showed an interhelical distance of $12 \AA$ and a packing volume of $16.1 \mathrm{~nm}^{3}$, and the second lowest energy structure II revealed an interhelical distance of $10 \AA$ and a volume of $15.0 \mathrm{~nm}^{3}$ (Figure 7B). The modeled EpoR-T242N TM dimer also exhibited two low-energy interfaces (Figure 7A). However, in contrast to the EpoR TM dimers the helices in both structures revealed a decrease in packing density with an interhelical distance of $14 \AA$ and a packing volume of 17.0 and $17.1 \mathrm{~nm}^{3}$, respectively (Figure 7B). Both low-energy structures for EpoR and EpoR-T242N were found to be lefthanded helix -helix pairs with a crossing angle of $20^{\circ}$.

In contrast to EpoR-T242N, the EpoR TM asparagine mutants L240N, L241N, V243N, L244N, and A245N surrounding position 242 , which were detectable in vesicle-like structures comparable to wild-type EpoR (Figure 1B and Figure S5 of the Supporting Information), displayed at least one structure with high packing density of the respective TM dimers (Figure 7B and Figures S5 and S6 of the Supporting Information). Of these L241N and A245N showed only one lowest energy structure exhibiting a particularly small interhelical distance as well as packing volume. Interestingly, these receptor variants are remarkably efficient in activating signal transduction (Figure 3) and eliciting biological re- 
A
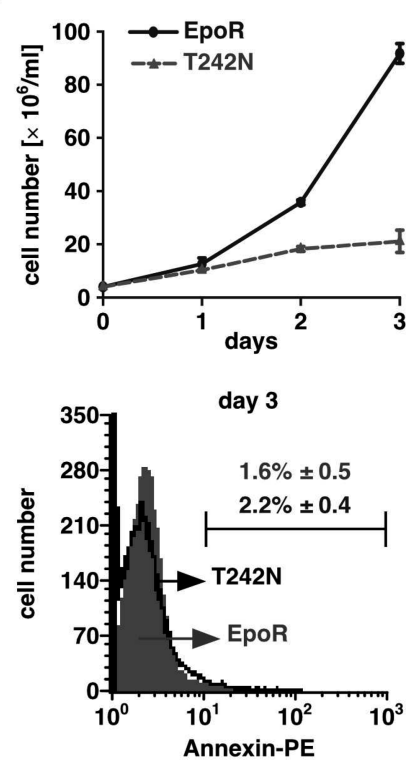

B

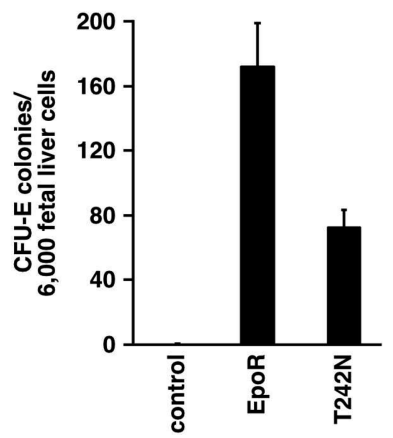

FIGURE 6: Proliferation and differentiation analysis of cells expressing EpoR-T242N. (A) BaF3 cells stably expressing HA-tagged wildtype EpoR or EpoR-T242N were cultivated for 3 days in 1 unit/ $\mathrm{mL}$ Epo. Cell numbers were determined each day (mean $\pm \mathrm{SD}, n$ $=3$ ). As control, cells cultivated in 5\% IL-3 conditioned medium counted in parallel displayed equal cell numbers (data not shown) (upper panel). BaF3 cells taken from cultures described above were stained with annexin V-PE and 7-AAD. Representative results are displayed for 3 days of cultivation as an overlay gated on 7-AAD negative cells, and the fraction of apoptotic cells is given as the percentage of annexin $\mathrm{V}$ positive cells (mean $\pm \mathrm{SD}, n=3$ ) (lower panel). (B) For reconstitution of EpoR ${ }^{-1-}$ CFU-E colonies, fetal liver cells from d12.5 $\mathrm{EpoR}^{-1-}$ mice were transduced with HAtagged wild-type EpoR or EpoR-T242N, grown for 2 days, and stained with benzidine. Mock-transduced cells were taken as control. Dark blue colonies larger than eight cells/colony were counted as CFU-E colonies, and their number was determined for two cell pools and expressed as mean $\pm \mathrm{SD}(n=3)$.

sponses (22) (Figure S4 of the Supporting Information) despite severely or slightly reduced cell surface expression of EpoR-L241N and EpoR-A245N, respectively (Figure 1A).

Correlating Packing Density of the EpoR TM Domain with Amplification of Signaling. To design additional mutants with an increased or decreased interhelical distance compared to wild-type EpoR, we applied the molecular model focusing on T242 in the EpoR TM domain. Comparable to asparagine, glutamine is a strong hydrogen donor and acceptor whereas alanine represents an apolar residue. Molecular modeling of the respective EpoR TM mutants EpoR-T242Q and EpoRT242A revealed one low-energy structure each. The EpoRT242Q TM dimer exhibited an interhelical distance of $14 \AA$ and a packing volume of $17.0 \mathrm{~nm}^{3}$ (Figure $8 \mathrm{~A}$ and Figure
A EpoR TM dimer:

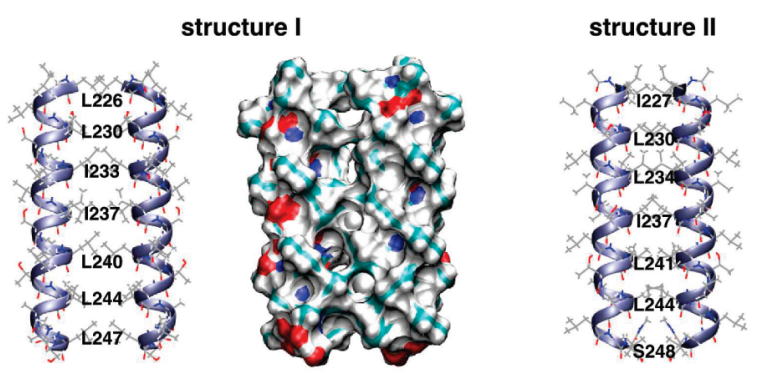

T242N TM dimer:

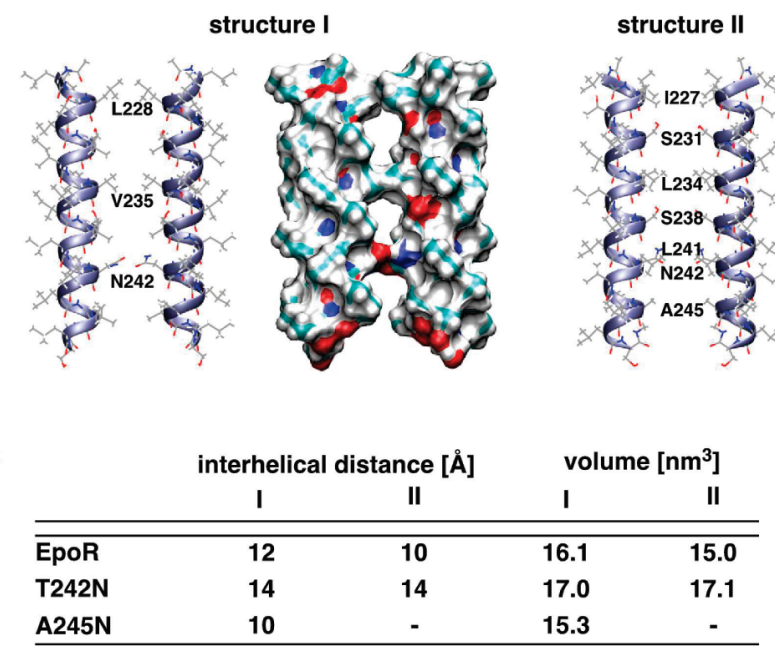

FIgURE 7: All-atom structures of EpoR TM dimers modeled in a membrane environment. (A) The lowest energy (structure I) and second lowest energy structures (structure II) of wild-type EpoR (upper panel) and EpoR-T242N (lower panel) TM dimers are depicted as ribbon diagrams (front view), and amino acids lining the helical interface are indicated. For structure II of the EpoRT242N TM dimer, N242 is also marked. For structure I, the solventaccessible surface area (front view) is shown in addition. (B) The values for interhelical distance and volume are given for the wildtype EpoR TM dimers as well as mutant EpoR TM dimers T242N and $\mathrm{A} 245 \mathrm{~N}$.

S6 of the Supporting Information) equal to the EpoR-T242N TM dimer, while the EpoR-T242A TM dimer displayed an interhelical distance of $10 \AA$ and a packing volume of 15.6 $\mathrm{nm}^{3}$.

Flow cytometry analysis showed decreased levels of cell surface expression for EpoR-T242Q but similar levels for EpoR-T242A in comparison to wild-type EpoR (Figure 8B). Examination of the subcellular localization of GFP-tagged EpoR-T242Q and EpoR-T242A in NIH3T3 cells revealed that whereas vesicle-like structures were detectable for EpoRT242A similar to wild-type EpoR, these structures were absent in EpoR-T242Q expressing cells (Figure 8C) resembling EpoR-T242N.

The activation of signaling pathways by EpoR-T242Q and EpoR-T242A was examined in $\mathrm{BaF} 3$ cells. Comparable to EpoR-T242N, receptor activation as well as phosphorylation of ERK1/2 and Akt/PKB through EpoR-T242Q was dramatically reduced, whereas activation of STAT5 at elevated Epo levels was similar to that of wild-type EpoR (Figure 9A). Though cell surface expression of EpoR-T242Q was reduced relative to wild-type EpoR (Figure 8B), this cannot entirely account for inefficient signaling since EpoR-L241N, which is expressed at the plasma membrane to similar levels 


\begin{tabular}{lcccc} 
& \multicolumn{2}{c}{ interhelical distance $[\AA]$} & \multicolumn{2}{c}{ volume $\left[\mathrm{nm}^{3}\right]$} \\
& I & I & I & \| \\
\hline \hline EpoR & 12 & 10 & 16.1 & 15.0 \\
T242N & 14 & 14 & 17.0 & 17.1 \\
T242Q & 14 & - & 17.0 & - \\
T242A & 10 & - & 15.6 & - \\
\hline
\end{tabular}

B

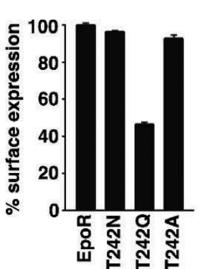

C
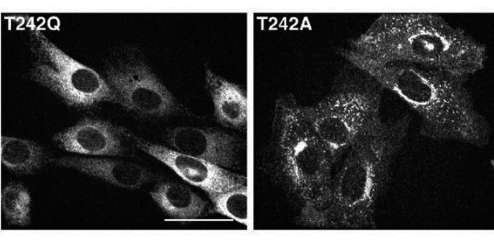

FIGURE 8: Correlation of all-atom structures of EpoR TM dimers and live cell imaging data. (A) The values for interhelical distance and volume are given for all-atom structures of the wild-type EpoR TM dimers and the mutant EpoR TM dimers T242N, T242Q, and T242A. (B) Cell surface expression of GFP-tagged wild-type HAEpoR and HA-EpoR TM domain mutants in stably transduced BaF3 cells was assessed by flow cytometry using a rat anti-HA antibody followed by a Cy5-coupled anti-rat IgG antibody. Live cells were gated using forward and side scatter, and cell surface expression is shown as the percentage of the wild-type EpoR (mean $\pm \mathrm{SD}, n=$ 3). (C) Expression of GFP-tagged wild-type HA-EpoR or HA-EpoR TM domain mutants in NIH3T3 cells was analyzed by spinning disk confocal microscopy, and representative images are shown. Scale bar is $20 \mu \mathrm{m}$.

as EpoR-T242Q, is nevertheless capable of mediating efficient signaling at elevated Epo levels (22). Interestingly and in line with dense TM helix packing, at low ligand concentrations of $1 \mathrm{unit} / \mathrm{mL}$ Epo, receptor activation and phosphorylation of STAT5, ERK1/2, and Akt/PKB were more pronounced in $\mathrm{BaF} 3$ cells expressing EpoR-T242A compared to wild-type EpoR expressing cells (Figure 9A).

Correlating Packing Volume of the EpoR TM Domain with Biological Responsiveness. To investigate the effect of altered packing density of the EpoR TM domain on biological responses, we analyzed Epo-dependent proliferation of $\mathrm{BaF} 3$ cells expressing GFP-tagged EpoR variants. Whereas BaF3EpoR-T242N and BaF3-EpoR-T242Q cells exhibited a dramatically reduced cell number compared to wild-type receptor expressing $\mathrm{BaF} 3$ cells (Figure 9B), interestingly BaF3-EpoR-T242A cells showed significantly better proliferative capacities at lower ligand concentrations of 0.1 and 0.5 unit $/ \mathrm{mL}$ Epo as revealed by a two-sided unpaired Student's $t$ test (Figure 9B). Furthermore, the capacity to support proliferation and differentiation of erythroid progenitor cells was examined by expressing GFP-tagged EpoR variants in fetal liver cells from $\mathrm{EpoR}^{-1-}$ embryos. As expected, mock-transduced fetal liver cells formed no CFU-E colonies (Figure 9C), whereas transduction of wild-type EpoR resulted in the formation of $48 \pm 6$ colonies. In contrast, reconstitution with EpoR-T242N or EpoR-T242Q revealed reduced numbers of $28 \pm 5$ or $30 \pm 6$ CFU-E colonies, respectively. Remarkably, expression of the mutant EpoR-T242A resulted in a significantly increased number of $61 \pm 5$ CFU-E colonies. Statistical relevance was confirmed by a two-sided unpaired Student's $t$ test (Figure 9C). Thus, compared to wild-type EpoR decreased packing density of the TM domain correlates with reduced capacities to support biological responses whereas tighter packing density is linked to enhanced responsiveness.

\section{DISCUSSION}

In this study, we demonstrated that dense packing of the EpoR TM domain promotes ligand-dependent responses of erythroid progenitor cells. Moreover, we could show that modulating the packing density of the TM helices allows for selective amplification of signaling cascades emanating from the EpoR and influences specific cellular decisions.

Determinants and motifs that regulate the assembly of TM helices have been assessed by several experimental approaches (28). By applying the ToxR assay, which is based upon insertion of chimeric proteins into the inner bacterial membrane, it has been shown that the EpoR TM possesses a strong capacity to self-interact (11). This ability contributes to maximal signaling responses (11) and can be enhanced by substitution of asparagine residues at specific positions (22). The experimental setup of the ToxR assay permitted determination of the region being most important for selfinteraction of the TM domain. Unexpectedly, analysis of EpoR TM mutants in the context of the full-length receptor showing the highest tendency of self-assembly in the ToxR assay revealed no evidence for constitutive receptor activation (data not shown) (22). However, in this context the insertion of bulky side chains such as asparagine is likely to introduce structural changes of the TM helix that might prevent efficient signaling. Therefore, molecular modeling of TM domain interactions is essential to predict functional implications of mutations within the TM domain and to gain a deeper insight into the relationship of the TM domain structure and the onset of signaling of dimeric cell surface receptors.

So far, structural data on full-length signaling receptors are unavailable. Therefore, we believe that a modeling approach is helpful to gain further insight into initial signaling events at the receptor level. Thus, to determine the effect of mutations on the structure of the EpoR TM domain dimer, we applied an implicit five-slab membrane model (16) that allows exposure of $\mathrm{OH}$ groups on the molecular surface of a helix dimer. In line with our model that identifies L226 or I227 as residues lining the two low-energy interfaces of the wild-type EpoR TM dimer, recent evidence showed that independent replacement of these two residues by cysteine results in constitutively active EpoR receptor variants $(29,30)$. Additionally, the two interfaces of the wild-type EpoR TM dimer we identified by all-atom modeling were both reported to be functionally active in biological assays applying receptor variants in which the extracellular domain of the EpoR has been substituted by a coiled-coil Put3 domain (15). This finding implies a model for EpoR activation in which binding of the ligand may stabilize the dynamic interaction of energetically favorable receptor dimers and thereby kinetically enable the initiation of signal transduction. Modeling in vacuum by Seubert et al. (15) identified two clusters that have been proposed to correspond to a functionally active as well as inactive interface. The discrepancy to our results could be due to the difference in considering the membrane environment as we include in our five-slab model. Currently, structural data for the EpoR are only available for the extracellular domain based on crystallographic analysis (7). Values of $73 \AA$ for free receptor and $39 \AA$ for ligand-occupied receptor indicated by Livnah et al. (7) reflect the distance of subdomains in the extracellular domain close to the cell membrane but not specifically the TM domain of 
A

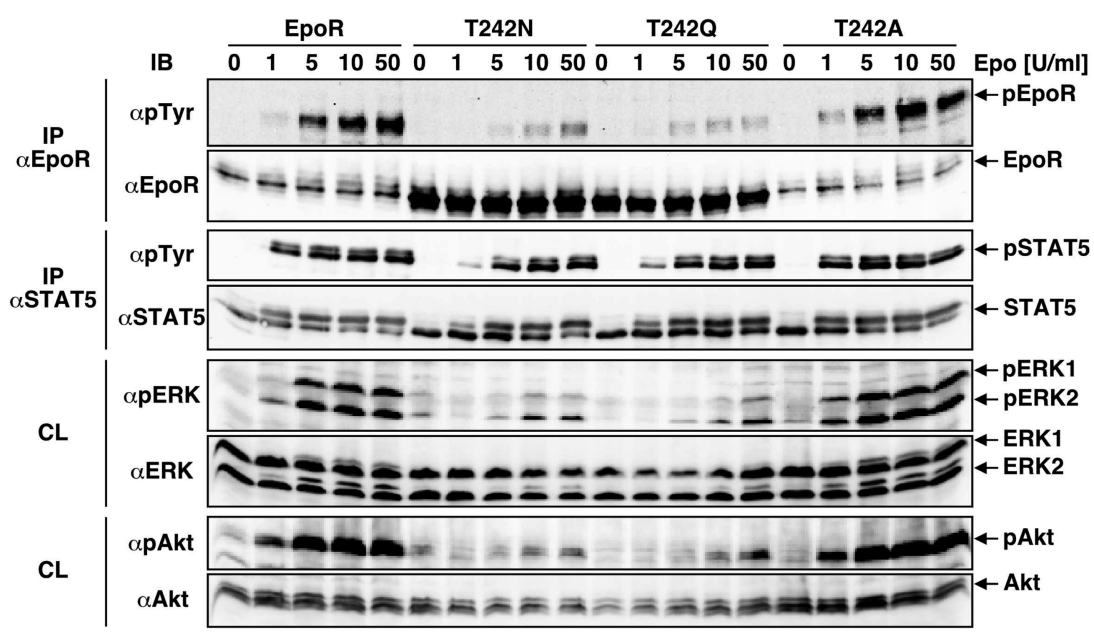

B
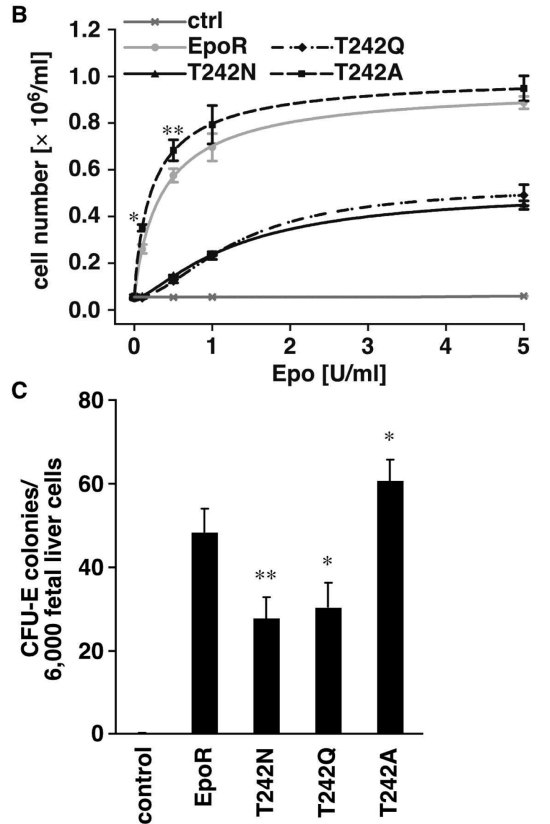

FIGURE 9: Immunoblot analysis and biological responsiveness of EpoR TM domain mutants. (A) BaF3 cells stably expressing GFP-tagged wild-type HA-EpoR or HA-EpoR mutants were stimulated with increasing Epo concentrations for 10 min. Immunoblots (IB) of cellular lysates (CL) were analyzed with anti-phospho-Akt, anti-phospho-ERK, anti-Akt, and anti-ERK antibodies. Immunoprecipitations (IP) with anti-EpoR and anti-STAT5 antibodies were analyzed by immunoblotting with anti-phosphotyrosine (pTyr) antibody, and membranes were reprobed with anti-EpoR or anti-STAT5 antibodies. Immunoblot signals were acquired using a charge-coupled device camera. The experiment was performed three times, and a representative immunoblot analysis is shown. (B) BaF3 cells stably expressing GFP-tagged wild-type HA-EpoR or HA-EpoR mutants were cultivated in medium supplemented with rising concentrations of Epo or without Epo. Mock-transduced cells were taken as control (ctrl). After 4 days, cell numbers were determined by Coulter counter (mean $\pm \mathrm{SD}, n=3$ ). Significance was calculated with a two-sided unpaired Student's $t$ test comparing wild-type EpoR and EpoR-T242A (*, $p<0.05 ; * *, p<0.01$ ). (C) For reconstitution of EpoR ${ }^{-1-}$ CFU-E colonies, fetal liver cells isolated from d12.5 EpoR ${ }^{-1-}$ mice were transduced with GFP-tagged wild-type HA-EpoR or HA-EpoR mutants, and mock-transduced cells were taken as control. Cells were grown for 2 days and stained with benzidine. Dark blue colonies larger than eight cells/colony were counted as CFU-E colonies, and their number was determined for two cell pools and expressed as mean $\pm \mathrm{SD}(n=3)$. Significance was calculated with a two-sided unpaired Student's $t$ test comparing EpoR TM domain mutants with wild-type EpoR (*,p<0.05; **, $p<0.01)$.

the EpoR and therefore do not necessarily contradict the distances observed in our modeling approach.

Further molecular modeling analysis revealed a decreased packing density of the TM helices of EpoR-T242N as well as EpoR-T242Q. In contrast, the mutant EpoR-T242A showed increased packing density of its TM domain compared to the lowest energy structure of the wild-type EpoR TM dimer. In addition to a decreased packing density of the EpoR-T242N TM dimers, the helices of the lowest energy structure are hydrogen-bonded by asparagine. Besides glutamine, glutamic acid, and aspartic acid, inclusion of asparagine in TM helices can promote strong interhelical association (31). Interhelical hydrogen bonding of the EpoRT242N TM dimer in our model suggests that the effects detected in the ToxR assay may reflect a stabilization of the dimeric form of the EpoR-T242N.

The mutant EpoR-T242N reveals processing and internalization kinetics comparable to wild-type EpoR, although vesicle-like structures including receptor-positive endosomes were not detectable by live cell imaging for EpoR-T242N and EpoR-T242Q in contrast to wild-type EpoR as well as EpoR-T242A. Since internalization rates show no difference for wild-type EpoR and EpoR-T242N, it is unlikely that endosomal signaling is a major cause of impaired signaling through EpoR-T242N, and we have no evidence that wildtype EpoR colocalizing with endosomal markers correlates with the activated form of the receptor. Since previous reports indicated that theEpoR can form dimers and oligomers $(12,32,33)$, we speculate that dynamic formation of oligomeric structures facilitates the detection of fluorescently tagged EpoR in transport vesicles, whereas oligomerization of EpoR-T242N is impaired and vesicle-like structures are no longer detectable. Previously, it was suggested that clustering of the EpoR after dimethyl sulfoxide pretreatment mediates amplification of biological responsiveness in erythroleukemia cells (34). Since it has been shown that dimethyl sulfoxide changes the structure of the lipid bilayer (35), this treatment could promote higher order oligomerization mediated by dynamic interaction of the EpoR TM domain. Cross-linking studies with [ $\left.{ }^{125} \mathrm{I}\right]$-Epo have not revealed clear evidence for higher oligomeric structures $(36,37)$, but this could be due to the choice of the cross-linker and low levels of EpoR at the cell surface.

Signaling through the EpoR promotes proliferation, survival, and differentiation of erythroid progenitor cells (10). Based on in vivo mouse experiments it has been proposed that STAT5 controls survival of erythroid cells $(38,39)$. We observe that EpoR-T242N efficiently activates STAT5 and promotes survival of the hematopoietic cell line $\mathrm{BaF} 3$ despite reduced levels of receptor activation. In accordance with these results, analysis of EpoR activation by the erythropoiesis-stimulating protein NESP (darbepoetin alfa) showed that STAT5 activation is robust even at suboptimal levels of receptor phosphorylation (40). Despite STAT5 activation, both EpoR-T242N and EpoR-T242Q are strongly impaired in activating ERK1 and Akt/PKB, key components of the 
MAPK and PI3K signaling cascades, respectively. This directly correlates with reduced proliferative and differentiation capacities of these mutant receptor variants in erythroid progenitors. Furthermore, recent studies show that STAT5 regulates iron uptake of erythroid cells through control of transferrin receptor 1 transcription $(41,42)$. Accordingly and in line with comparable STAT5 activation by wild-type EpoR and EpoR-T242N, CFU-E colonies although formed to a lesser extent showed no visible reduction of benzidine-stained hemoglobin for EpoR-T242N compared to wild-type EpoR (data not shown). Since cell surface expression, half-life of the mature cell surface form, and internalization rates are comparable for wild-type EpoR and EpoR-T242N, packing density of the TM is the major difference correlating with impaired signaling through ERK1/2 and Akt/PKB and therefore with impaired biological responsiveness. However, further structural changes might be additionally introduced when replacing threonine by other amino acids such as asparagine, glutamine, or alanine.

In contrast, the mutant EpoR-T242A exhibits a higher packing density of the TM dimers and shows enhanced activation of the receptor as well as of the ERK1/2 and Akt/ PKB at low levels of Epo stimulation. This hypersensitivity to ligand stimulation results in a higher biological responsiveness compared to wild-type EpoR as revealed by the capacity to support the formation of erythroid progenitor cells at the CFU-E stage. Epo-mediated PI3K signaling has been shown to promote maturation of erythroid progenitor cells (43), and activation of Akt/PKB signaling has been furthermore linked to tissue-protective effects of Epo in neuronal cells (44). By exploiting the predictive power of our molecular modeling approach, we could show that elevated packing density of the EpoR TM domain translates to increased potency to drive proliferation and differentiation at low levels of ligand concentration. Thus, we report a murine EpoR mutant based on a single amino acid substitution that is still Epo-dependent but shows enhanced biological responsiveness compared to the wild-type receptor at low Epo levels. In human primary polycythemia, mutations of the EpoR gene affecting a single residue are rather rare (45). Instead, a defect on the level of receptor signaling is most commonly related to frameshift mutations leading to truncated receptors unable to associate withnegative regulators (46-49).

The amino acid sequence corresponding to L240-A245 of the murine EpoR is conserved in different mammalian species but, interestingly, with the exception of the threonine residue. Unlike murine, rat, and human EpoR, the sequence of the TM domain of dog, pig, sheep, bovine, and zebu exhibits an alanine residue instead (Figure 10). We speculate that the natural occurrence of alanine in the EpoR TM domain of this phylogenetically distinct group of mammalian species (50) might constitute a level to regulate the extent of erythropoiesis especially at low steady-state levels of in vivo Epo stimulation or a high demand of erythrocyte renewal.

In conclusion, we could show that a high packing density of the EpoR TM domain facilitates selective amplification of signaling pathways as well as biological responses. This concept may be applicable to other cytokine receptors that have been reported to form preformed dimers. To our knowledge, this is the first report of a mutation resulting not in a constitutively active but in a signaling-optimized

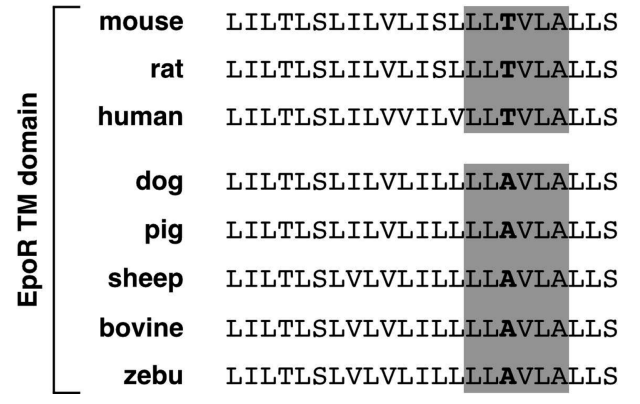

FIgURE 10: Amino acid sequences of potential EpoR TM domains of different mammalian species. The region encompassing the mutant TM dimers analyzed by molecular modeling for the murine EpoR is highlighted in gray, and the amino acids corresponding to the murine receptor residue T242 are depicted in bold letters. The UniProtKB/Swiss-Prot accession numbers are P14753 (mouse), Q07303 (rat), P19235 (human), Q2KL21 (dog), and Q9MYZ9 (pig), and the UniProtKB/TrEMBL accession numbers are Q95N13 (sheep), Q28206 (bovine), and Q27950 (zebu).

cytokine receptor solely based on a single amino acid substitution. In analogy to the computational selection of synthetic peptides for intervention of integrin signaling (17), the establishment of a molecular model that successfully permits the correlation of TM domain packing density with biological responsiveness provides an important tool for the rational design of therapeutic components targeting hematological disorders.

\section{ACKNOWLEDGMENT}

We thank Dr. Stefan Constantinescu, Dr. Marino Zerial, Dr. Roger Tsien, and Dr. Hong Wu for providing reagents. We also thank Dr. Jennifer Reed and Dr. Dieter Langosch for critical discussions. Finally, we thank the Nikon Imaging Center at the University of Heidelberg for providing the spinning disk confocal microscope and Dr. Ulrike Engel for expert advice.

\section{SUPPORTING INFORMATION AVAILABLE}

Supporting materials and methods, supporting results, and supporting figures: colocalization of wild-type EpoR and endosomal markers (Figure S1), analysis of cell surfacebound $\left[{ }^{125} \mathrm{I}\right]$-Epo (Figure S2), coimmunoprecipitation of activated receptor with JAK2 and SHP-1 (Figure S3), proliferation analysis of cells expressing EpoR-A245N (Figure S4), live cell imaging and all-atom modeling of additional EpoR TM domain mutants (Figure S5), and allatom models of mutant EpoR TM dimers (Figure S6). This material is available free of charge via the Internet at http:// pubs.acs.org.

\section{REFERENCES}

1. Jiang, G., and Hunter, T. (1999) Receptor signaling: When dimerization is not enough. Curr. Biol. 9, R568-R571.

2. Bargmann, C. I., Hung, M. C., and Weinberg, R. A. (1986) Multiple independent activations of the neu oncogene by a point mutation altering the transmembrane domain of p185. Cell 45, 649-657.

3. Longo, N., Shuster, R. C., Griffin, L. D., Langley, S. D., and Elsas, L. J. (1992) Activation of insulin receptor signaling by a single amino acid substitution in the transmembrane domain. J. Biol. Chem. 267, 12416-12419.

4. Forbes, L. V., Gale, R. E., Pizzey, A., Pouwels, K., Nathwani, A., and Linch, D. C. (2002) An activating mutation in the transmembrane domain of the granulocyte colony-stimulating factor receptor in patients with acute myeloid leukemia. Oncogene 21, 5981-5989. 
5. Onishi, M., Mui, A. L., Morikawa, Y., Cho, L., Kinoshita, S., Nolan, G. P., Gorman, D. M., Miyajima, A., and Kitamura, T. (1996) Identification of an oncogenic form of the thrombopoietin receptor MPL using retrovirus-mediated gene transfer. Blood 88, 1399-1406

6. Ding, J., Komatsu, H., Wakita, A., Kato-Uranishi, M., Ito, M., Satoh, A., Tsuboi, K., Nitta, M., Miyazaki, H., Iida, S., and Ueda, R. (2004) Familial essential thrombocythemia associated with a dominant-positive activating mutation of the c-MPL gene, which encodes for the receptor for thrombopoietin. Blood 103, 41984200.

7. Livnah, O., Stura, E. A., Middleton, S. A., Johnson, D. L., Jolliffe, L. K., and Wilson, I. A. (1999) Crystallographic evidence for preformed dimers of erythropoietin receptor before ligand activation. Science 283, 987-990.

8. Gent, J., van Kerkhof, P., Roza, M., Bu, G., and Strous, G. J. (2002) Ligand-independent growth hormone receptor dimerization occurs in the endoplasmic reticulum and is required for ubiquitin systemdependent endocytosis. Proc. Natl. Acad. Sci. U.S.A. 99, 98589863.

9. Devos, R., Guisez, Y., Van der Heyden, J., White, D. W., Kalai, M., Fountoulakis, M., and Plaetinck, G. (1997) Ligand-independent dimerization of the extracellular domain of the leptin receptor and determination of the stoichiometry of leptin binding. J. Biol. Chem. 272, 18304-18310.

10. Wu, H., Liu, X., Jaenisch, R., and Lodish, H. F. (1995) Generation of committed erythroid BFU-E and CFU-E progenitors does not require erythropoietin or the erythropoietin receptor. Cell 83,59 67.

11. Kubatzky, K. F., Ruan, W., Gurezka, R., Cohen, J., Ketteler, R., Watowich, S. S., Neumann, D., Langosch, D., and Klingmüller, U. (2001) Self assembly of the transmembrane domain promotes signal transduction through the erythropoietin receptor. Curr. Biol. $11,110-115$.

12. Constantinescu, S. N., Keren, T., Socolovsky, M., Nam, H., Henis, Y. I., and Lodish, H. F. (2001) Ligand-independent oligomerization of cell-surface erythropoietin receptor is mediated by the transmembrane domain. Proc. Natl. Acad. Sci. U.S.A. 98, 4379-4384.

13. Fleishman, S. J., Schlessinger, J., and Ben-Tal, N. (2002) A putative molecular-activation switch in the transmembrane domain of erbB2. Proc. Natl. Acad. Sci. U.S.A. 99, 15937-15940.

14. Kim, S., Chamberlain, A. K., and Bowie, J. U. (2003) A simple method for modeling transmembrane helix oligomers. J. Mol. Biol. 329, 831-840.

15. Seubert, N., Royer, Y., Staerk, J., Kubatzky, K. F., Moucadel, V., Krishnakumar, S., Smith, S. O., and Constantinescu, S. N. (2003) Active and inactive orientations of the transmembrane and cytosolic domains of the erythropoietin receptor dimer. Mol. Cell 12, 12391250.

16. Sengupta, D., Meinhold, L., Langosch, D., Ullmann, G. M., and Smith, J. C. (2005) Understanding the energetics of helical peptide orientation in membranes. Proteins 58, 913-922.

17. Yin, H., Slusky, J. S., Berger, B. W., Walters, R. S., Vilaire, G., Litvinov, R. I., Lear, J. D., Caputo, G. A., Bennett, J. S., and DeGrado, W. F. (2007) Computational design of peptides that target transmembrane helices. Science 315, 1817-1822.

18. Remy, I., Wilson, I. A., and Michnick, S. W. (1999) Erythropoietin receptor activation by a ligand-induced conformation change. Science 283, 990-993.

19. Richmond, T. D., Chohan, M., and Barber, D. L. (2005) Turning cells red: signal transduction mediated by erythropoietin. Trends Cell Biol. 15, 146-155.

20. Ketteler, R., Glaser, S., Sandra, O., Martens, U. M., and Klingmüller, U. (2002) Enhanced transgene expression in primitive hematopoietic progenitor cells and embryonic stem cells efficiently transduced by optimized retroviral hybrid vectors. Gene Ther. 9, 477-487.

21. Ketteler, R., Heinrich, A. C., Offe, J. K., Becker, V., Cohen, J., Neumann, D., and Klingmüller, U. (2002) A functional green fluorescent protein-tagged erythropoietin receptor despite physical separation of JAK2 binding site and tyrosine residues. J. Biol. Chem. 277, 26547-26552.

22. Ruan, W., Becker, V., Klingmüller, U., and Langosch, D. (2004) The interface between self-assembling erythropoietin receptor transmembrane segments corresponds to a membrane-spanning leucine zipper. J. Biol. Chem. 279, 3273-3279.

23. Klingmüller, U., Lorenz, U., Cantley, L. C., Neel, B. G., and Lodish, H. F. (1995) Specific recruitment of SH-PTP1 to the erythropoietin receptor causes inactivation of JAK2 and termination of proliferative signals. Cell 80, 729-738.

24. Leach, A. R. (2001) Molecular modelling: principles and applications, 2nd ed., Pearson Education EMA, Harlow, Essex.

25. Branden, C., and Tooze, J. (1999) Introduction to protein structure, 2nd ed., Garland Science, New York.

26. Canutescu, A. A., Shelenkov, A. A., and Dunbrack, R. L., Jr. (2003) A graph-theory algorithm for rapid protein side-chain prediction. Protein Sci. 12, 2001-2014.

27. Brooks, B. R., Bruccoleri, R. E., Olafson, B. D., States, D. J., Swaminathan, S., and Karplus, M. (1983) CHARMM: A program for macromolecular energy, minimization, and dynamics calculation. J. Comput. Chem. 4, 187-217.

28. Arkin, I. T. (2002) Structural aspects of oligomerization taking place between the transmembrane alpha-helices of bitopic membrane proteins. Biochim. Biophys. Acta 1565, 347-363.

29. Kubatzky, K. F., Liu, W., Goldgraben, K., Simmerling, C., Smith, S. O., and Constantinescu, S. N. (2005) Structural requirements of the extracellular to transmembrane domain junction for erythropoietin receptor function. J. Biol. Chem. 280, 14844-14854.

30. Lu, X., Gross, A. W., and Lodish, H. F. (2006) Active conformation of the erythropoietin receptor: random and cysteine-scanning mutagenesis of the extracellular juxtamembrane and transmembrane domains. J. Biol. Chem. 281, 7002-7011.

31. Zhou, F. X., Cocco, M. J., Russ, W. P., Brunger, A. T., and Engelman, D. M. (2000) Interhelical hydrogen bonding drives strong interactions in membrane proteins. Nat. Struct. Biol. 7, 154160 .

32. McCaffery, P. J., Fraser, J. K., Lin, F. K., and Berridge, M. V. (1989) Subunit structure of the erythropoietin receptor. J. Biol. Chem. 264, 10507-10512.

33. Miura, O., and Ihle, J. N. (1993) Dimer- and oligomerization of the erythropoietin receptor by disulfide bond formation and significance of the region near the WSXWS motif in intracellular transport. Arch. Biochem. Biophys. 306, 200-208.

34. Yonekura, S., Chern, Y., Donahue, K. A., Feldman, L., Vanasse, G. J., and Sytkowski, A. J. (1991) Erythropoietin receptors induced by dimethyl sulfoxide exhibit positive cooperativity associated with an amplified biologic response. Proc. Natl. Acad. Sci. U.S.A. 88, 2535-2539.

35. Gordeliy, V. I., Kiselev, M. A., Lesieur, P., Pole, A. V., and Teixeira, J. (1998) Lipid membrane structure and interactions in dimethyl sulfoxide/water mixtures. Biophys. J. 75, 2343-2351.

36. Damen, J., Mui, A. L., Hughes, P., Humphries, K., and Krystal, G. (1992) Erythropoietin-induced tyrosine phosphorylations in a high erythropoietin receptor-expressing lymphoid cell line. Blood 80, 1923-1932.

37. Takahashi, T., Chiba, S., Hirano, N., Yazaki, Y., and Hirai, H. (1995) Characterization of three erythropoietin (Epo)-binding proteins in various human Epo-responsive cell lines and in cells transfected with human Epo-receptor cDNA. Blood 85, 106-114.

38. Socolovsky, M., Fallon, A. E., Wang, S., Brugnara, C., and Lodish, H. F. (1999) Fetal anemia and apoptosis of red cell progenitors in Stat $5 \mathrm{a}-/-5 \mathrm{~b}-/-$ mice: a direct role for Stat 5 in $\mathrm{Bcl}-\mathrm{X}(\mathrm{L})$ induction. Cell 98, 181-191.

39. Menon, M. P., Karur, V., Bogacheva, O., Bogachev, O., Cuetara, B., and Wojchowski, D. M. (2006) Signals for stress erythropoiesis are integrated via an erythropoietin receptor-phosphotyrosine-343Stat5 axis. J. Clin. Invest. 116, 683-694.

40. Elliott, S., Egrie, J., Browne, J., Lorenzini, T., Busse, L., Rogers, N., and Ponting, I. (2004) Control of rHuEPO biological activity: the role of carbohydrate. Exp. Hematol. 32, 1146-1155.

41. Kerenyi, M. A., Grebien, F., Gehart, H., Schifrer, M., Artaker, M., Kovacic, B., Beug, H., Moriggl, R., and Mullner, E. W. (2008) Stat 5 regulates cellular iron uptake of erythroid cells via IRP-2 and TfR-1. Blood (in press).

42. Zhu, B. M., McLaughlin, S. K., Na, R., Liu, J., Cui, Y., Martin, C., Kimura, A., Robinson, G. W., Andrews, N. C., and Hennighausen, L. (2008) Hematopoietic-specific Stat5-null mice display microcytic hypochromic anemia associated with reduced transferrin receptor gene expression. Blood 112, 2071-2080.

43. Ghaffari, S., Kitidis, C., Zhao, W., Marinkovic, D., Fleming, M. D., Luo, B., Marszalek, J., and Lodish, H. F. (2006) AKT induces erythroid-cell maturation of JAK2-deficient fetal liver progenitor cells and is required for Epo regulation of erythroid-cell differentiation. Blood 107, 1888-1891.

44. Chong, Z. Z., Kang, J. Q., and Maiese, K. (2003) Erythropoietin fosters both intrinsic and extrinsic neuronal protection through 
modulation of microglia, Akt1, Bad, and caspase-mediated pathways. Br. J. Pharmacol. 138, 1107-1118.

45. Le Couedic, J. P., Mitjavila, M. T., Villeval, J. L., Feger, F., Gobert, S., Mayeux, P., Casadevall, N., and Vainchenker, W. (1996) Missense mutation of the erythropoietin receptor is a rare event in human erythroid malignancies. Blood 87, 1502-1511.

46. de la Chapelle, A., Traskelin, A. L., and Juvonen, E. (1993) Truncated erythropoietin receptor causes dominantly inherited benign human erythrocytosis. Proc. Natl. Acad. Sci. U.S.A. 90, 4495-4499.

47. Sokol, L., Luhovy, M., Guan, Y., Prchal, J. F., Semenza, G. L., and Prchal, J. T. (1995) Primary familial polycythemia: a frameshift mutation in the erythropoietin receptor gene and increased sensitivity of erythroid progenitors to erythropoietin. Blood 86, 15-22.
48. Arcasoy, M. O., Degar, B. A., Harris, K. W., and Forget, B. G. (1997) Familial erythrocytosis associated with a short deletion in the erythropoietin receptor gene. Blood 89, 4628-4635.

49. Furukawa, T., Narita, M., Sakaue, M., Otsuka, T., Kuroha, T., Masuko, M., Azegami, T., Kishi, K., Takahashi, M., Utsumi, J., Koike, T., and Aizawa, Y. (1997) Primary familial polycythaemia associated with a novel point mutation in the erythropoietin receptor. Br. J. Haematol. 99, 222-227.

50. Bininda-Emonds, O. R., Cardillo, M., Jones, K. E., MacPhee, R. D., Beck, R. M., Grenyer, R., Price, S. A., Vos, R. A., Gittleman, J. L., and Purvis, A. (2007) The delayed rise of present-day mammals. Nature 446, 507-512.

BI801425E 